\title{
The synchronized trial on expectant mothers with depressive symptoms by omega-3 PUFAs (SYNCHRO): Study protocol for a randomized controlled trial
}

Daisuke Nishi ${ }^{1,2,3^{*}}$ (D), Kuan-Pin Su ${ }^{4,5^{*}}$, Kentaro Usuda ${ }^{1,2}$, Yi-Ju Jill Chiang ${ }^{4}$, Tai-Wei Guu ${ }^{4}$, Kei Hamazaki ${ }^{6}$, Naoki Nakaya ${ }^{7}$, Toshimasa Sone ${ }^{8}$, Yo Sano ${ }^{9}$, Yoshiyuki Tachibana ${ }^{10}$, Hiroe Ito ${ }^{1}$, Keiich Isaka ${ }^{1}$, Kenji Hashimoto ${ }^{11}$, Tomohito Hamazaki ${ }^{12}$ and Yutaka J Matsuoka ${ }^{1,13}$

\begin{abstract}
Background: Maternal depression can be harmful to both mothers and their children. Omega-3 polyunsaturated fatty acid (PUFA) supplementation has been investigated as an alternative intervention for pregnant women with depressive symptoms because of the supporting evidence from clinical trials in major depression, the safety advantage, and its anti-inflammatory and neuroplasticity effects. This study examines the efficacy of omega-3 PUFA supplementation for pregnant women with depressive symptoms in Taiwan and Japan, to provide evidence available for Asia. The rationale and protocol of this trial are reported here.

Methods: The Synchronized Trial on Expectant Mothers with Depressive Symptoms by Omega-3 PUFAs (SYNCHRO) is a multicenter, double-blind, parallel group, randomized controlled trial. Participants will be randomized to either the omega-3 PUFAs arm (1,200 mg eicosapentaenoic acid and $600 \mathrm{mg}$ docosahexaenoic acid daily) or placebo arm. Primary outcome is total score on the Hamilton Rating Scale for Depression (HAMD) at 12 weeks after the start of the intervention. We will randomize 56 participants to have $90 \%$ power to detect a 4.7-point difference in mean HAMD scores with omega-3 PUFAs compared with placebo. Because seafood consumption varies across countries and this may have a major effect on the efficacy of omega-3 PUFA supplementation, 56 participants will be recruited at each site in Taiwan and Japan, for a total number of 112 participants. Secondary outcomes include depressive symptoms at 1 month after childbirth, diagnosis of major depressive disorder, changes in omega-3 PUFAs concentrations and levels of biomarkers at baseline and at 12 weeks' follow-up, and standard obstetric outcomes. Data analyses will be by intention to treat. The trial was started in June 2014 and is scheduled to end in February 2018.
\end{abstract}

Discussion: The trial is expected to provide evidence that can contribute to promoting mental health among mothers and children in Asian populations.

Trial registration: Clinicaltrials.gov: NCT02166424. Registered 15 June 2014; University Hospital Medical Information Network (UMIN) Center: UMIN000017979. Registered 20 May 2015.

Keywords: Omega-3 polyunsaturated fatty acids, Eicosapentaenoic acid, Depression, Pregnancy, Prevention

\footnotetext{
*Correspondence: d-nishi@umin.ac.jp; cobolsu@gmail.com

'Department of Obstetrics and Gynecology, Tokyo Medical University, 6-7-1

Nishi-shinjuku, Shinjuku-ku, Tokyo 160-0023, Japan

${ }^{4}$ Department of Psychiatry \& Mind-Body Interface Laboratory (MBI-Lab), China

Medical University Hospital, No. 2, Yuh-Der Road, Taichung 404, Taiwan

Full list of author information is available at the end of the article
} 


\section{Background}

A systematic review reported the prevalence of depression during pregnancy was $7.4 \%$ for the first trimester, $12.8 \%$ for the second, and $12.0 \%$ for the third [1]. A meta-analysis estimated the prevalence of major and minor depression in the range of 6.5 to $12.9 \%$ during the different trimesters of pregnancy and during the first 12 months postpartum [2]. Depression during pregnancy can have harmful effects on both the mother and child. The mother may experience difficulties performing daily activities, fail to seek prenatal care, have a poor diet, use tobacco, alcohol, or other harmful substances, and be at risk of self-harm or suicide [3]; fetal growth rate may be slower; and the child may have temperament or behavioral problems later [4-6].

The established treatment options for depression include antidepressants, cognitive behavioral therapy (CBT), and interpersonal psychotherapy (IPT). However, there are some limitations to each of these options. In terms of antidepressants, guidelines recommend all antidepressant drugs be used with caution during pregnancy and that selective serotonin reuptake inhibitors such as paroxetine be avoided [7, 8]. In fact, it was found in a population screening study of pregnant women attending antenatal clinics that only $11 \%$ of pregnant women with major depressive disorders (MDD) were actually receiving suitable antidepressant medication [9]. Both CBT and IPT are recommended for pregnant women with mild or moderate depression [10] and an RCT has shown that IPT was effective for depression during pregnancy [11], but pregnant women cannot always access CBT or IPT. Identifying a safe alternative treatment strategy for depression during pregnancy is therefore desirable.

Many meta-analyses of RCTs [12-19], although not all $[20,21]$, support the positive effects of omega-3 polyunsaturated fatty acid (omega-3 PUFA) supplementation on depressive symptoms. The latest evidence supports the efficacy of omega-3 PUFAs rich in eicosapentaenoic acid (EPA) against depression [14, 16-18].

Omega-3 PUFAs are essential nutrients for maintaining physiological function of the mothers and infants during pregnancy. A previous study showed that omega3 PUFA content in the brain of pregnant rats can be reduced after a single reproductive cycle when they are deprived of sufficient dietary omega-3 PUFAs, a reduction which may affect neuronal function [22]. In addition, another study reported that the brain decreases in volume in pregnant women [23]. It has been suggested that changes in the phospholipid content of the brain's membranes could reduce brain size [24]. It is known that the composition of maternal phospholipid membranes can be altered during pregnancy by the fetus scavenging essential fatty acids such as omega-3 PUFAs, and this could potentially change brain morphology [23]. A lack of omega-3 PUFAs, then, may have a harmful effect on maternal mental health as well as on neurodevelopment of the fetus and infant. A naturalistic longitudinal study showed that lower seafood intake in pregnant women was associated with an increased risk of their children having suboptimum neurodevelopment [25]. To date, although two RCTs have failed to show the efficacy of omega-3 PUFAs for pregnant women with depression $[26,27]$, the supplements used contained high levels of docosahexaenoic acid (DHA) rather than EPA.

In a previous RCT conducted in Taiwan, our group showed the efficacy of supplements with a high ratio of EPA (2.2 g daily) to DHA (1.2 g daily) for depression in pregnancy [28]. However, it is not yet clear what the appropriate amount of supplemental omega-3 PUFAs is and it may differ between countries because of national differences in the consumption of fish, which is a rich source of omega-3 PUFAs. Although fish consumption in Taiwan is higher than in most Western countries, it is half that in Japan [29]. The recommended daily intake for pregnant women in Japan is $1.8 \mathrm{~g}$ of omega-3 PUFAs [30], and the usual dose of pure EPA as a prescription drug is also set at $1.8 \mathrm{~g}$ daily.

Recently, we suggested the potential efficacy of moderate amount of omega-3 PUFAs supplementation for improving depressive symptoms in our open label trial conducted in Japan and Taiwan [31]. The objective of the present randomized controlled trial is to determine the efficacy of a daily dose of $1.8 \mathrm{~g}$ of omega-3 PUFAs supplements (1.2 g EPA and $0.6 \mathrm{~g}$ DHA) for depressive symptoms in pregnant women in Japan and Taiwan. Examining the efficacy and safety of $1.8 \mathrm{~g}$ of omega-3 PUFAs supplementation in different countries is clinically relevant because not only is fish consumption generally higher and the prevalence of depression generally lower in East Asian countries such as Japan and Taiwan compared with Western countries, but also there are considerable differences between East Asian countries [29]. The study is called the Synchronized Trial on Expectant Mothers with Depressive Symptoms by Omega-3 PUFAs (SYNCHRO), and this paper explains the rationale and describes protocol of the trial. An additional file provides the completed SPIRIT 2013 Checklist (see Additional file 1).

\section{Methods/Design}

SYNCHRO is a multicenter, double-blind, parallel group, randomized controlled trial that will allocate participants to an intervention arm to receive omega-3 fatty acid supplementation or a parallel placebo arm in the ratio of 1:1. The trial will run from June 2014 through February 2018, with participant enrollment between June 2014 and August 2017. 
The study was registered at clinicaltrials.gov (NCT0 2166424) on June 15, 2014 and the University Hospital Medical Information Network (UMIN) Center (UMIN0 00017979) on June 22, 2015.

\section{Inclusion criteria}

Pregnant women who are between 12 and 24 weeks of gestation will be recruited and followed through their pregnancy and up to 4 weeks after childbirth. The inclusion criteria are:

1) Pregnant and aged $\geq 20$ years

2) Japanese conversational ability at the Japan site or Chinese conversational ability at the Taiwan site to ensure participants have sufficient understanding of the trial's scope and can provide written informed consent

3) Planning to remain in the Tokyo area or Taichung area for 4-6 weeks after childbirth

4) Edinburgh Postnatal Depression Scale (EPDS) score of $\geq 9$

5) In good physical health as judged by an obstetrician

\section{Exclusion criteria}

The presence of any of the following will result in exclusion from the trial.

1) History or current suspicion of psychosis, bipolar I disorder, substance abuse or related disorder, eating disorder, or personality disorder

2) Serious psychiatric symptoms, such as self-harm behavior, or in need of rapid psychiatric treatment

3) Normal birth not expected (e.g., fetal malformation)

4) History of bleeding disorder such as von Willebrand's Disease

5) Regular treatment with aspirin or warfarin for the past 3 months

6) Smoking habit of $\geq 40$ cigarettes per day

7) Regular treatment with ethyl icosapentate or regular consumption of omega-3 PUFA supplements for the past 3 months

8) Habit of eating fish as a main dish $\geq 4$ times per week

\section{Procedure}

Eligible patients will be screened using the EPDS at three locations in cooperation with obstetricians: Toda Chuo Women's Hospital and the National Center for Child Health and Development, both in Japan, and China Medical University Hospital, Taiwan. Toda Chuo Women's Hospital is a local base hospital, and the National Center for Child Health and Development and China Medical University Hospital are academic hospitals. Clinical research coordinators, who will be welltrained nurses, psychologists or psychiatrists, will invite eligible patients to take part in the study. Patients will receive a comprehensive obstetric examination before the study commences and undergo a baseline assessment before randomization. Figure 1 shows a flow diagram of the study protocol.

\section{Randomization}

Central registration involved assigning participants to each group according to an assignment diagram that was developed by a statistician. Using a computergenerated random allocation sequence, they created block randomization tables with two stratification factors: diagnosis of current MDD and study site. The test will be double-blinded and only after all participants have completed the protocol and all the results have been tabulated will the research term be informed of the results of the randomization. Stratification was justified by previous studies: the results of a meta-analysis showed some evidence supporting the benefits of omega-3 PUFAs in individuals diagnosed with MDD but no evidence supporting any benefits in individuals without MDD [32]. Moreover, a previous study showed that seafood consumption predicted prevalence rates of postpartum depression across countries [33].

\section{Intervention}

Participants will take omega-3 PUFAs capsules or placebo capsules for 12 weeks, and can take an entire day's dosage at once or spread throughout the day. The omega-3 PUFAs capsules have been formulated to each contain $134 \mathrm{mg}$ of EPA and $67.7 \mathrm{mg}$ of DHA, and a total of 9 capsules containing a total of $1206 \mathrm{mg}$ EPA and $609 \mathrm{mg}$ DHA will be administered daily. The placebo capsules have been formulated to contain $320 \mathrm{mg}$ of olive oil and $9.9 \mathrm{mg}$ of omega-3 fatty acids. Trace amounts of semi-deodorized fish oil are added to the control oil so that its smell is not perceivably different from that of the active oil.

Adherence to the regimens will be checked by clinical research coordinators. Whole blood samples $(7 \mathrm{ml})$ collected with ethylene-diamine tetraacetic acid will be taken at baseline, at 12 weeks' follow-up, and at 1 month after childbirth and omega-3 PUFAs will be determined as described previously [34]. Briefly, erythrocytes will be washed two time with saline and stored at $-80{ }^{\circ} \mathrm{C}$ until analysis. The method of Bligh and Dyer [35] will be used to extract total lipids, and total phospholipid fractions will be separated by thin-layer chromatography and transmethylated with by treatment with a mixture of $\mathrm{HCl}$ and methanol. Next, fatty acid composition will be analyzed by gas chromatography (GC-2014 Shimadzu Corporation, Kyoto, Japan) controlled with GC-solution version 2.3 (Shimadzu Corporation, Kyoto, Japan) and equipped with a DB-225 capillary column (length, $30 \mathrm{~m}$; internal diameter, $0.25 \mathrm{~mm}$; film $0.25 \mu \mathrm{m}$; J\&M Scientific, Folsom, CA). 


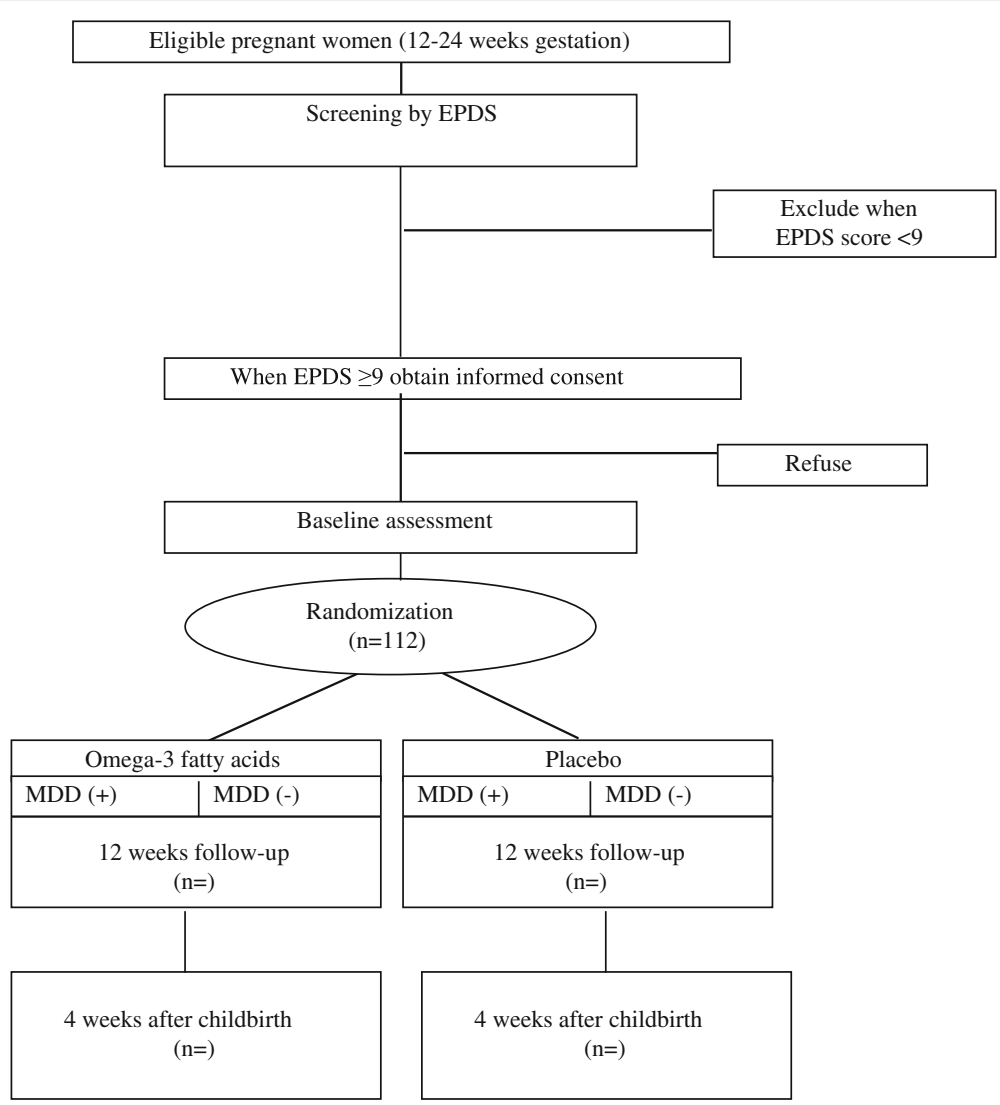

Fig. 1 Flow diagram of the study. EPDS: Edinburgh Postnatal Depression Scale; MDD: major depressive disorders

\section{Assessment}

The assessment schedule from baseline to 1 month after childbirth is shown in Table 1.

\section{Outcome measures}

\section{Primary outcome}

The primary outcome is total score on the 17 -item Hamilton Rating Scale for Depression (HAMD) [36, 37] at 12 weeks after the start of the intervention. HAMD is a widely used structural interview for assessment of depressive symptoms and has been used during pregnancy and in the postpartum period [26, 28, 38]. Trained psychiatrists, psychologists, or nurses will conduct the structured interviews for the evaluation. To assess inter-rater reliability, 3 well-trained raters in Japan and 2 well-trained raters in Taiwan have independently assessed the same person acting as a patient in a video. The original video was made in Japanese and then translated into Chinese for the raters in Taiwan. The maximum variance in scores among the 5 raters from the expert gold standard score was satisfactory for all the HAMD items. The intraclass correlation coefficient for the total HAMD score among the 5 raters was 0.967 (95\% confidence interval, 0.934-0.986).
Table 1 Outcome measures and assessments

\begin{tabular}{|c|c|c|c|}
\hline & Baseline & 12 weeks & 1 month after childbirth \\
\hline \multicolumn{4}{|l|}{ Primary outcome } \\
\hline HAMD & + & + & + \\
\hline \multicolumn{4}{|l|}{ Secondary outcome } \\
\hline MINI & + & + & + \\
\hline EPDS & + & + & + \\
\hline BDI-II & + & + & + \\
\hline Plasma & + & + & + \\
\hline Serum & + & + & + \\
\hline \multicolumn{4}{|l|}{ Other measures } \\
\hline Demographics & + & & \\
\hline Obstetric outcomes & & & + \\
\hline Erythrocyte & + & + & + \\
\hline Adverse effects & & + & + \\
\hline
\end{tabular}

HAMD hamilton rating scale for depression (HAMD), MINI mini international neuropsychiatric interview, EPDS Edinburgh postnatal depression scale, BDI-II beck depression inventory-II 


\section{Secondary outcomes}

The secondary outcomes are:

- Total HAMD score at 4 weeks after childbirth

- Major depressive disorder (MDD) as determined by the depression module of the Mini International Neuropsychiatric Interview (MINI) [39] at 12 weeks after starting the intervention and at 4 weeks after childbirth-MINI is a concise structural interview for the major Axis I psychiatric disorders described in the Diagnostic and Statistical Manual of Mental Disorders, Fourth Edition and the International Classification of Diseases and Related Health Problems, Tenth Revision

- Total scores on the EPDS [40] at 12 weeks after starting the intervention and at 4 weeks after childbirth-EPDS is a widely used 10-item self-report scale to assess the severity of perinatal depressive symptoms in the past week; EPDS scores $>9$ may indicate the presence of MDD [41]

- Beck Depression Inventory II (BDI-II) [42] at 12 weeks after starting the intervention and at 4 weeks after childbirth-BDI-II is a widely used 21-item self-report scale to measure the severity of depressive symptoms in the past 2 weeks [42]; it was not designed to perinatal mood specifically but has been used during pregnancy and in the postpartum period [28, 43]

- Biomarkers levels at baseline, 12 weeks' follow-up, and 1 month after childbirth for

- Omega-3 fatty acids concentrations in erythrocytes

- Brain-derived neurotrophic factor (BDNF) in serum

- Estrogen in plasma

- Oxytocin in plasma

- Progesterone in plasma

- Human chorionic gonadotropin in plasma

- Phospholipase A2 in plasma

For the measurement of biomarkers, plasma and serum will be drawn at baseline, at 12 weeks' follow-up, and at 1 month after childbirth. Samples will be stored in separate freezers at $-80{ }^{\circ} \mathrm{C}$ until analysis. All samples will be analyzed on the same day and under the same conditions by a biochemist blinded to the severity of depressive symptoms and participants' diagnostic status.

\section{Other outcomes}

Standard obstetric outcomes will be analyzed: gestational age, gestational diabetes mellitus, gestational hypertension or preeclampsia, induced labor, estimated blood loss, cesarean section, operative vaginal delivery, birthweight, 1-min and 5-min Apgar scores, and neonatal intensive care unit admission. Data will be collected on discontinuation of the allocated interventions.

\section{Participant protection and reporting of adverse events}

We define an adverse event as any unwanted or unintended sign, symptom, or disease observed in the trial participants, whether or not it is caused by study intervention. Safety issues will be monitored by interview, telephone, or email. Researchers and clinical research coordinators will record and evaluate any suspected adverse events. The follow criteria will guide evaluation of the severity of adverse [34].

1) Mild: Some symptoms or signs observed. Treatment is not required for the participant to continue in the trial.

2) Moderate: Some symptoms or signs observed. The participant may reduce the dose of test capsules or receive treatment such as additional drugs to continue the trial.

3) Severe: Clinical symptoms are severe enough to interfere with daily activities. Discontinuation of test capsules may be necessary.

The following are always regarded as serious adverse events, regardless of test dosage.

1) An event that results in death

2) A life-threatening event

3) An event that requires hospitalization (or extends an existing hospitalization period) in order to provide treatment

4) An event that results in a permanent or prominent disorder or a failure in body function

5) An event that results in a subsequent congenital anomaly or deficiency

6) Other events considered medically significant

Any serious adverse event will be reported to and assessed by the Data and Safety Monitoring Board as soon as possible. The ethics committee will also receive the report. Other adverse events will be reported and evaluated regularly. Information on concomitant drug use and reason for use will also be reported.

\section{Estimation of sample size}

The expected difference in total HAMD score between the two arms at 12 weeks' follow-up is set at 4.7 according to a previous study [28]. It is expected that $12 \%$ of participants will discontinue the test capsules or drop out of the trial. Given an $\alpha$ level of 0.05 (two-tailed), a $\beta$ level of 0.10 , and standard deviation of 5 , the desired number of participants will be 28 in each arm and 56 in total. Because a previous study suggested that seafood 
consumption varied across countries [33] and this may have a major effect on the efficacy of omega-3 PUFAs, 56 participants will be recruited at each site in Japan and Taiwan. Thus, the total number of participants will be 112. However, it is possible that enrollment will be halted before reaching this target if significant financial or logistical factors arise, or that number of cases at one of the sites may exceed the target number depending on the accumulation of cases at the other site.

\section{Statistical analysis \\ Primary analysis}

Primary analysis will be conducted according to the intention-to-treat principle. Mean score differences, $95 \%$ confidence intervals, and $\mathrm{P}$ values will be calculated using analysis of covariance in order to examine whether participants receiving omega-3 PUFAs supplementation score on average 4.7 points lower on the HAMD at 12 weeks' follow-up than participants receiving placebo. Two-tailed tests will be used, with the $\alpha$ level set at $5 \%$. The assignment remains masked, but any background factors that may predict primary outcome will be entered as covariates if such factors are found.

\section{Exploratory analysis}

We will conduct exploratory analysis of secondary outcomes and sub-groups and therefore multiplicity will not be controlled. A mixed effect model with repeated measures will be evaluated by regression. The validity of the findings will be examined through sensitivity analysis, including per protocol analysis and analysis of the method used to impute missing variables.

\section{Ethical considerations}

This study protects the rights and welfare of participants in the spirit of ethical guidelines outlined under the Declaration of Helsinki. Informed consent to participate in the study will be obtained from all participants. Personal information about potential and enrolled participants will be strictly secured to avoid external leaks before, during, and after the trial. No special compensation will be paid in the event of health damage directly related to the research. The research plan was deliberated on and approved by the following ethics committees: Tokyo Medical University, Japan, 26 June 2013; and China Medical University, Taiwan, 6 December 2013. If the protocol needs to be changed for any reason, the principal investigators will communicate this with the institutional review boards.

\section{Data and safety monitoring}

The data manager will use a premade template to create a report to be sent to the principal investigator and the
Data and Safety Monitoring Board once every 6-12 months. The members of the Data and Safety Monitoring Board are Professor Koichiro Watanabe (psychopharmacology), Dr. Katsumi Ikeshita (clinical psychiatrist), and Dr. Tomohiro Nakao (psychotherapy), who are professionals with clinical trial experience with no involvement in the trial. The monitoring report includes the following.

1) Progress of the study and case registration

2) Psychiatric evaluation status and any relevant issues

3) Any adverse events

4) Discussion of other relevant issues

\section{Discontinuing intervention}

Intervention will be discontinued if the participant does not wish to continue taking the trial substance or placebo, or if continuing the intervention would be difficult because medically required concomitant medication may be affected by the trial substance. Subsequent evaluations and follow-up will proceed as designed.

\section{Presentation of the trial results}

The findings of the trial will be presented in medical journals and at academic conferences. If particularly noteworthy results are obtain or the study is accepted by an influential academic publication, an application for a press release will be made. The principal investigators are, in general, listed as corresponding authors. The order of presentation of the first author and co-authors will be determined according to their intellectual contributions. Individuals who join the study after its approval and work toward implementing and conducting the study may obtain authorship pending approval by the principal investigators.

\section{Discussion}

The findings of the SYNCHRO study are expected to influence decisions on the clinical care of pregnant women with depressive symptoms. The strengths of the study include its double-blind, placebo-controlled design, its multicenter, multi-country collaboration, and its evaluation of an EPA-rich supplement.

Maternal depression can have a significant harmful influence on both mothers and children. Due to the possibility of adverse effects of antidepressants, omega-3 PUFAs may provide a safe alternative for pregnant women with depressive symptoms. So far, only 2 RCTs have examined the efficacy of omega-3 PUFAs with a high ratio of EPA for depression in pregnancy [28, 43]; one of which recruited pregnant women without current depression but with a history of depression [43]. In addition, the result of one study on omega-3 PUFAs conducted in one country may not be able to be applicable to other countries due to differences in fish 
consumption [33]. The SYNCHRO study should provide evidence for two different populations in Asia and contribute to promoting mental health of large numbers of mothers and children.

\section{Trial status}

This randomized trial is now enrolling participants and conducting follow-up.

\section{Additional file}

Additional file 1: SPIRIT Checklist. (DOC $124 \mathrm{~kb}$ )

\section{Abbreviations}

BDI-II: Beck depression inventory II; BDNF: Brain-derived neurotrophic factor; CBT: Cognitive behavioral therapy; DHA: Docosahexaenoic acid; EPA: Eicosapentaenoic acid; EPDS: Edinburgh postnatal depression scale; HAMD: Hamilton rating scale for depression; IPT: Interpersonal psychotherapy; MDD: Major depressive disorders; MINI: Mini international neuropsychiatric interview; PUFA: Omega-3 polyunsaturated fatty acid; RCT: Randomized controlled trial

\section{Acknowledgements}

The authors thank Hisateru Tachimori Ph.D., for his technical assistance with preparing allocated supplements.

\section{Funding}

This work was supported by Grant-in-Aid for Young Scientists (A) from the Japan Society for the Promotion of Science (25702043) and Intramural Research Grant (27-3-2) for Neurological and Psychiatric Disorders of National Center of Neurology and Psychiatry, Japan (to DN) and from the Taiwan Ministry of Science and Technology and China Medical University (to KPS). The funding body peer reviewed proposal documents which included a digest version of this study protocol as part of the selection process. All of the supplements used in the study were supplied by Kentech Co, Ltd., Toyama, Japan, which had no role in the study design and conduct, in the collection, analysis and interpretation of the data, or in the preparation, review, and approval of the manuscript.

\section{Availability of data and materials}

Drs. Daisuke Nishi and Kuan-Pin Su have full access to all of the data in the study and take responsibility for the integrity of the data and the accuracy of analysis.

Because we did not plan for the dataset to be shared when making the study protocol, we have not obtained consent from the study participants to share the data. Thus, the data will not be shared.

\section{Authors' contributions}

DN and KPS are principal investigators and obtained funding. DN, KPS and YJM conceived of the study and drafted the original protocol. $\mathrm{KH}$ (Kei Hamazaki), KH (Kenji Hashimoto) and TH contributed to the refinement of the protocol. NN and TS decided the analytic strategy. DN, KPS, KU, YJC and TWG managed the enrollment procedure. YS, YT, HI and $\mathrm{KI}$ contributed to administrative and technical development. All authors have read and approved the final manuscript.

\section{Authors' information}

"Not applicable" in this section.

\section{Competing interests}

Dr. Nishi has received research grants from the Japan Society for the Promotion of Science, the National Center of Neurology and Psychiatry Japan, the Ministry of Health, Labour and Welfare, and Japan Agency for Medical Research and Development, and a lecture fee from Otsuka Pharmaceutical Co., Ltd., and a manuscript fee from Sumitomo Dainippon Pharma Co., Ltd. Dr. Su has received research grants from the Taiwan Ministry of Science and Technology and China Medical University. Dr. Kei
Hamazaki received research support from an Intramural Research Grant for Neurological and Psychiatric Disorders from the National Center of Neurology and Psychiatry and a grant from the Japan Society for the Promotion of Science; and scholarship donations from Otsuka Pharmaceutical Co., Ltd. Dr. Tomohito Hamazaki has received lecture fees from Takeda Pharmaceutical Co., Ltd. and travel expenses from Aker BioMarine. Dr. Matsuoka has received an Intramural Research Grant for Neurological and Psychiatric Disorders from the National Center of Neurology and Psychiatry Japan and a grant from Pfizer Health Research Foundation, as well as lecture fees from Ono Pharmaceutical Co., Ltd., Mochida Pharmaceutical Co., Ltd., Takeda Pharmaceutical Company Ltd., Suntory Wellness Ltd., DHA \& EPA Association, and Otsuka Pharmaceutical Co., Ltd. No other authors report any financial disclosures.

\section{Consent for publication}

"Not applicable" in this section.

\section{Ethics approval and consent to participate}

This study protects the rights and welfare of participants in the spirit of ethical guidelines outlined under the Declaration of Helsinki. The research plan was deliberated on and approved by the ethics committees of Tokyo Medical University, Japan (26 June 2013) and China Medical University, Taiwan (6 December 2013). Informed consent to participate in the study will be obtained from all participants.

\section{Author details}

'Department of Obstetrics and Gynecology, Tokyo Medical University, 6-7-1 Nishi-shinjuku, Shinjuku-ku, Tokyo 160-0023, Japan. ${ }^{2}$ National Institute of Mental Health, National Center of Neurology and Psychiatry, 4-1-1 Ogawahigashicho, Kodaira, Tokyo 187-8553, Japan. ${ }^{3}$ Department of Public Mental Health Policy, Graduate School of Medicine, The University of Tokyo, 4-1-1, Ogawahigashicyo, Kodaira, Tokyo 187-8553, Japan. ${ }^{4}$ Department of Psychiatry \& Mind-Body Interface Laboratory (MBI-Lab), China Medical University Hospital, No. 2, Yuh-Der Road, Taichung 404, Taiwan. ${ }^{5}$ Graduate Institute of Neural and Cognitive Sciences, College of Medicine, China Medical University, No.91, Hsueh-Shih Road, Taichung 404, Taiwan. ${ }^{6}$ Department of Public Health, Faculty of Medicine, University of Toyama, 2630 Sugitani, Toyama, Toyama 930-0194, Japan. ${ }^{7}$ Department of Preventive Medicine and Epidemiology, Tohoku Medical Megabank Organization, Tohoku University, 2-1 Seiryo-machi, Aoba-ku, Sendai, Miyagi 980-8573, Japan. ${ }^{8}$ Department of Rehabilitation, Faculty of Health Science, Tohoku Fukushi University, 6-149-1 Kunimigaoka, Aoba-ku, Sendai, Miyagi 989-3201, Japan. ${ }^{9}$ Toda Chuo Women's Hospital, 2-26-3 Kamitoda, Toda, Saitama 335-0022, Japan. ${ }^{10}$ Division of Infant and Toddler Mental Health, Department of Psychosocial Medicine, National Center for Child Health and Development, 2-10-1 Ookura, Setagaya-ku, Tokyo 157-8535, Japan. ${ }^{11}$ Division of Clinical Neuroscience, Chiba University Center for Forensic Mental Health, 1-8-1 Inohana, Chiba 260-8670, Japan. ${ }^{12}$ Department of Medicine, Toyama Jonan Onsen Daini Hospital, 1-13-6, Taromarunishimachi, Toyama, Toyama 939-8271, Japan. ${ }^{13}$ Division of Health Care Research, Center for Public Health Sciences, National Cancer Center (YJM), 5-1-1 Tsukiji, Chuo-ku, Tokyo 104-0045, Japan

Received: 18 August 2016 Accepted: 6 September 2016 Published online: 15 September 2016

\section{References}

1. Bennett HA, Einarson A, Taddio A, Koren G, Einarson TR. Prevalence of depression during pregnancy: systematic review. Obstet Gynecol. 2004; 103(4):698-709.

2. Gavin NI, Gaynes BN, Lohr KN, Meltzer-Brody S, Gartlehner G, Swinson T. Perinatal depression: a systematic review of prevalence and incidence. Obstet Gynecol. 2005;106(5 Pt 1):1071-83.

3. Yonkers KA, Wisner KL, Stewart DE, Oberlander TF, Dell DL, Stotland N, Ramin S, Chaudron L, Lockwood C. The management of depression during pregnancy: a report from the American Psychiatric Association and the American College of Obstetricians and Gynecologists. Gen Hosp Psychiatry. 2009:31(5):403-13.

4. Davis EP, Glynn LM, Schetter CD, Hobel C, Chicz-Demet A, Sandman CA. Prenatal exposure to maternal depression and cortisol influences infant temperament. J Am Acad Child Adolesc Psychiatry. 2007;46(6):737-46. 
5. Leech SL, Larkby CA, Day R, Day NL. Predictors and correlates of high levels of depression and anxiety symptoms among children at age 10. J Am Acad Child Adolesc Psychiatry. 2006;45(2):223-30.

6. Field T, Diego M, Hernandez-Reif M. Prenatal depression effects on the fetus and newborn: a review. Infant Behav Dev. 2006;29(3):445-55.

7. $\quad$ US Food and Drug Administration: Public health advisory: paroxetine. In.; Accessed 19 Sep 2012.

8. Medicine and Healthcare Products Regulatory Agency: MHRA update on the risks o birth defects in babies born to mothers taking paroxetine-q\&a. In.; Accessed 19 Sep 2012

9. Flynn HA, Blow FC, Marcus SM. Rates and predictors of depression treatment among pregnant women in hospital-affiliated obstetrics practices. Gen Hosp Psychiatry. 2006;28(4):289-95.

10. National Institute for Health and Clinical Excellence. Antenatal and potnatal mental health: the NICE guideline on clinical management and service guidance. London: National Institute for Health and Clinical Excellence; 2007.

11. Zlotnick C, Miller IW, Pearlstein T, Howard M, Sweeney P. A preventive intervention for pregnant women on public assistance at risk for postpartum depression. Am J Psychiatry. 2006;163(8):1443-5.

12. Freeman MP, Hibbeln JR, Wisner KL, Davis JM, Mischoulon D, Peet M, Keck Jr PE, Marangell LB, Richardson AJ, Lake J, et al. Omega-3 fatty acids: evidence basis for treatment and future research in psychiatry. J Clin Psychiatry. 2006;67(12):1954-67.

13. Ross BM, Seguin J, Sieswerda LE. Omega-3 fatty acids as treatments for mental illness: which disorder and which fatty acid? Lipids Health Dis. 2007;6:21.

14. Lin PY, Su KP. A meta-analytic review of double-blind, placebo-controlled trials of antidepressant efficacy of omega-3 fatty acids. J Clin Psychiatry. 2007;68(7):1056-61.

15. Martins JG. EPA but not DHA appears to be responsible for the efficacy of omega-3 long chain polyunsaturated fatty acid supplementation in depression: evidence from a meta-analysis of randomized controlled trials. J Am Coll Nutr. 2009;28(5):525-42.

16. Sublette ME, Ellis SP, Geant AL, Mann JJ. Meta-analysis of the effects of eicosapentaenoic acid (EPA) in clinical trials in depression. J Clin Psychiatry. 2011.

17. Martins JG, Bentsen H, Puri BK. Eicosapentaenoic acid appears to be the key omega-3 fatty acid component associated with efficacy in major depressive disorder: a critique of Bloch and Hannestad and updated meta-analysis. Mol Psychiatry. 2012

18. Lin PY, Mischoulon D, Freeman MP, Matsuoka Y, Hibbeln J, Belmaker RH, Su KP. Are omega-3 fatty acids antidepressants or just mood-improving agents? The effect depends upon diagnosis, supplement preparation, and severity of depression. Mol Psychiatry. 2012;17(12):1161-3. author reply 1163-1167.

19. Grosso G, Pajak A, Marventano S, Castellano S, Galvano F, Bucolo C, Drago F, Caraci F. Role of omega-3 fatty acids in the treatment of depressive disorders: a comprehensive meta-analysis of randomized clinical trials. PLoS ONE. 2014;9(5), e96905

20. Appleton KM, Hayward RC, Gunnell D, Peters TJ, Rogers PJ, Kessler D, Ness AR. Effects of n-3 long-chain polyunsaturated fatty acids on depressed mood: systematic review of published trials. Am J Clin Nutr. 2006;84(6):1308-16.

21. Bloch MH, Hannestad J. Omega-3 fatty acids for the treatment of depression: systematic review and meta-analysis. Mol Psychiatry. 2011

22. Levant B, Radel JD, Carlson SE. Reduced brain DHA content after a single reproductive cycle in female rats fed a diet deficient in N-3 polyunsaturated fatty acids. Biol Psychiatry. 2006;60(9):987-90.

23. Oatridge A, Holdcroft A, Saeed N, Hajnal JV, Puri BK, Fusi L, Bydder GM. Change in brain size during and after pregnancy: study in healthy women and women with preeclampsia. AJNR Am J Neuroradiol. 2002;23(1):19-26.

24. Horrobin DF. The membrane phospholipid hypothesis as a biochemical basis for the neurodevelopmental concept of schizophrenia. Schizophr Res. 1998;30(3):193-208.

25. Hibbeln JR, Davis JM, Steer C, Emmett P, Rogers I, Williams C, Golding J. Maternal seafood consumption in pregnancy and neurodevelopmental outcomes in childhood (ALSPAC study): an observational cohort study. Lancet. 2007:369(9561):578-85.

26. Rees AM, Austin MP, Parker GB. Omega-3 fatty acids as a treatment for perinatal depression: randomized double-blind placebo-controlled trial. Aust NZJ Psychiatry. 2008:42(3):199-205.

27. Makrides M, Gibson RA, McPhee AJ, Yelland L, Quinlivan J, Ryan P. Effect of DHA supplementation during pregnancy on maternal depression and neurodevelopment of young children: a randomized controlled trial. JAMA. 2010;304(15):1675-83.

28. Su KP, Huang SY, Chiu TH, Huang KC, Huang CL, Chang HC, Pariante CM. Omega-3 fatty acids for major depressive disorder during pregnancy: results from a randomized, double-blind, placebo-controlled trial. J Clin Psychiatry. 2008;69(4):644-51.

29. Hibbeln JR. Fish consumption and major depression. Lancet. 1998;351(9110):1213.

30. Japanese Ministry of Health LaW. Dietary Reference Intakes for Japanese (2015) for pregnant women and nursing mothers (in Japanese). 2014

31. Nishi D, Su KP, Usuda K, Chiang YJ, Guu TW, Hamazaki K, Nakaya N, Sone T, Sano $Y$, Ito $H$, et al. Omega-3 fatty acid supplementation for expectant mothers with depressive symptoms in Japan and Taiwan: An open-label trial. Psychiatry Clin Neurosci. 2016;70(6):253-4.

32. Appleton KM, Rogers PJ, Ness AR. Updated systematic review and metaanalysis of the effects of n-3 long-chain polyunsaturated fatty acids on depressed mood. Am J Clin Nutr. 2010;91(3):757-70.

33. Hibbeln JR. Seafood consumption, the DHA content of mothers' milk and prevalence rates of postpartum depression: a cross-national, ecological analysis. J Affect Disord. 2002;69(1-3):15-29.

34. Matsuoka Y, Nishi D, Yonemoto N, Hamazaki K, Matsumura K, Noguchi H, Hashimoto K, Hamazaki T. Tachikawa project for prevention of posttraumatic stress disorder with polyunsaturated fatty acid (TPOP): study protocol for a randomized controlled trial. BMC Psychiatry. 2013;13(1):8.

35. Bligh EG, Dyer WJ. A rapid method of total lipid extraction and purification. Can J Biochem Physiol. 1959;37(8):911-7.

36. Hamilton M. A rating scale for depression. J Neurol Neurosurg Psychiatry. 1960;23:56-62.

37. Williams JB, Kobak KA, Bech P, Engelhardt N, Evans K, Lipsitz J, Olin J, Pearson J, Kalali A. The GRID-HAMD: standardization of the Hamilton Depression Rating Scale. Int Clin Psychopharmacol. 2008;23(3):120-9.

38. Freeman MP, Davis M, Sinha P, Wisner KL, Hibbeln JR, Gelenberg AJ. Omega-3 fatty acids and supportive psychotherapy for perinatal depression: a randomized placebo-controlled study. J Affect Disord. 2008;110(1-2):142-8.

39. Sheehan DV, Lecrubier $Y$, Sheehan $K H$, Amorim $P$, Janavs J, Weiller $E$, Hergueta T, Baker R, Dunbar GC. The Mini-International Neuropsychiatric Interview (M.I.N.I.): the development and validation of a structured diagnostic psychiatric interview for DSM-IV and ICD-10. J Clin Psychiatry. 1998;59(20):22-33. quiz 34-57.

40. Cox JL, Holden JM, Sagovsky R. Detection of postnatal depression. Development of the 10-item Edinburgh Postnatal Depression Scale. Br J Psychiatry. 1987;150:782-6.

41. Okano T, Murata M, Masuji F, Tamaki R, Nomura J, Miyaoko H. Validation and reliability of Japanese version of the EPDS. Arch Psychiatr Diag Clin Evaluat. 1996;7(4):525-33.

42. Beck AT, Steer R, Brown GK. Manual for Beck Depression Inventory-II. San Antonio: Psychological Corporation; 1996.

43. Mozurkewich E, Chilimigras J, Klemens C, Keeton K, Allbaugh L, Hamilton S, Berman D, Vazquez D, Marcus S, Djuric Z, et al. The mothers, Omega-3 and mental health study. BMC Pregnancy Childbirth. 2011;11:46.

\section{Submit your next manuscript to BioMed Central and we will help you at every step:}

- We accept pre-submission inquiries

- Our selector tool helps you to find the most relevant journal

- We provide round the clock customer support

- Convenient online submission

- Thorough peer review

- Inclusion in PubMed and all major indexing services

- Maximum visibility for your research

Submit your manuscript at www.biomedcentral.com/submit
) Biomed Central 